\title{
Crosstalk between DGP branes
}

\author{
Rainer Dick ${ }^{1,2, a}$ \\ ${ }^{1}$ Department of Physics and Engineering Physics, University of Saskatchewan, 116 Science Place, Saskatoon, SK S7N 5E2, Canada \\ ${ }^{2}$ Perimeter Institute for Theoretical Physics, 31 Caroline Street North, Waterloo, ON N2L 2Y5, Canada
}

Received: 12 February 2015 / Accepted: 18 March 2015 / Published online: 26 March 2015

(c) The Author(s) 2015. This article is published with open access at Springerlink.com

\begin{abstract}
If two DGP branes carry $U(1)$ gauge theories and overlap, particles of one brane can interact with the photons from the other brane. This coupling modifies in particular the Coulomb potentials between charges from the same brane in the overlapping regions. The coupling also introduces Coulomb interactions between charges from the different branes which can generate exotic bound states. The effective modification of the fine structure constant in the overlap region generates a trough in signals at the redshift of the overlap region and an increase at smaller or larger redshift, depending on the value of the crosstalk parameter $g_{e} g_{p}$. This implies potentially observable perturbations in the Lyman $\alpha$ forest if our 3-brane overlapped with another 3 -brane in a region with redshift $z \lesssim 6$. Crosstalk can also affect structure formation by enhancing or suppressing radiative cooling.
\end{abstract}

\section{Introduction}

The idea of extra dimensions has been around in theoretical physics for almost a century $[1,2]$ and has been considerably expanded and reinvigorated in string theory. Furthermore, Dvali, Gabadadze, and Porrati (DGP) pointed out in 2000 that we could live in a higher-dimensional world with infinitely large extra dimensions hidden from plain sight because everything except gravity can only propagate on a 3 -brane in the higher-dimensional world [3,4]. The idea that observation of additional dimensions does not need to be suppressed by energy thresholds, but that instead there can be consistent restrictions of matter fields to submanifolds of a higher-dimensional universe was a significant advancement of our understanding of higher dimensions. Therefore, we denote a 3-brane carrying matter fields in an ambient spacetime with gravitational degrees of freedom as a DGP brane, including also e.g. 3-branes in cascading gravity mod-

a e-mail: rainer.dick@usask.ca els $[5,6]$. At this point we do not specify the background gravitational theory because we are interested in electromagnetic effects on the branes.

Shortly after the inception of DGP branes, it was pointed out that at least at the classical level they can support a modified Friedmann equation which may explain accelerated expansion without dark energy [7,8]. The stability of the self-accelerated solution has meanwhile been called into question $[9,10]$, but DGP branes can nevertheless support consistent modified cosmological evolution equations which comply with standard late time FLRW evolution [7, 8, 11-13]. On the other hand, it was found in $[11,12]$ and rediscovered in [14] that DGP branes can even support the standard Friedmann equation and all the corresponding standard cosmological models on the brane, i.e. the absence of cosmological signals from modified evolution equations does not rule out DGP branes. It is therefore important to also look for other possible experimental signatures of DGP branes.

In the present paper I would like to draw attention to the fact that overlap of DGP branes at or after reionization can generate perturbations in the Lyman $\alpha$ forest in the direction of the overlap region. This is based on the observation that particles from our brane can couple to photons from a $U(1)$ gauge theory on the second brane, thus impacting Coulomb interactions in the overlap region. This phenomenon of possible mixing of gauge interactions between two branes in an overlap region will be denoted as crosstalk. Indeed, it is possible and worthwhile to examine more general crosstalk models involving also Yukawa interactions between particles in overlapping brane volumes. We will focus on crosstalk interactions between charged particles and photons to study the impact of these interactions on electromagnetic potentials and the observed redshifts of spectral lines.

Crosstalk models, their impact on redshifts of spectral lines and consequences for the Lyman $\alpha$ forest are introduced in Sect. 2. Implications for structure formation and appearance of superlarge structures are outlined in Sect. 3, and the conclusions are summarized in Sect. 4. 


\section{Electromagnetic crosstalk between branes}

The action for fermions of masses $m_{I}$ and charges $q_{I}$ on our 3 -brane is

$$
\begin{aligned}
S_{1}[\psi, A]= & \int d^{4} x \mathcal{L}(\psi, A)=\int d^{4} x\left[-\frac{1}{4} F^{\mu v} F_{\mu \nu}\right. \\
& \left.+\sum_{I} \bar{\psi}_{I}\left(\mathrm{i} \gamma^{\mu} \partial_{\mu}+q_{I} \gamma^{\mu} A_{\mu}-m_{I}\right) \psi_{I}\right]
\end{aligned}
$$

if we can neglect curvature effects. We make the same assumption of approximate flatness for the second brane. At least weak curvature of at least one of the two branes will generically appear near the boundary of the overlap region, but we defer gravitational effects of overlapping branes for later studies. Here we are primarily interested in the effects of electromagnetic crosstalk in approximately flat regions of overlap.

A simple example of smooth overlap of two flat 3-branes is e.g. provided by a flat Minkowski 3-brane with inertial coordinates $x^{\mu}$ and perpendicular coordinate $\xi$ touched by a second brane with the same $t, y, z$ coordinates and the embedding into flat ambient five-dimensional spacetime given by

$\xi=\frac{1}{3 a^{2}} \Theta(x-a)(x-a)^{3} \pm \frac{1}{3 a^{2}} \Theta(-x-a)(x+a)^{3}$.

This 3-brane smoothly touches our Minkowski 3-brane for all values of $t, y, z$ and for $-a \leq x \leq a$. It actually smoothly penetrates through our 3-brane if we choose the plus sign in (2).

The induced metric on the second brane is

$$
\begin{aligned}
g_{\mu \nu}= & \eta_{\mu \nu}+\frac{1}{a^{4}} \eta_{\mu}^{1} \eta_{\nu}^{1}\left[\Theta(x-a)(x-a)^{4}\right. \\
& \left.+\Theta(-x-a)(x+a)^{4}\right]
\end{aligned}
$$

and vanishing of the Riemann tensor is easily verified. Of course, we can also simply introduce inertial coordinates on the second brane by defining $d X / d x=\sqrt{g_{x x}}$,

$$
\begin{aligned}
X= & \frac{1}{a^{2}} \Theta(-x-a)\left[\int_{0}^{x+a} d u \sqrt{a^{4}+u^{4}}-a^{3}\right] \\
& +\Theta\left(a^{2}-x^{2}\right) x \\
& +\frac{1}{a^{2}} \Theta(x-a)\left[\int_{0}^{x-a} d u \sqrt{a^{4}+u^{4}}+a^{3}\right] .
\end{aligned}
$$

We are generically interested in finite volume overlaps, and then we do have to allow for at least weak curvature on the boundaries of the overlap region, but this example and infinitely many similar examples demonstrate that 3-branes can smoothly overlap, share segments of their geodesics in the overlap region, and yet be separately geodesically complete. We will adapt the DGP framework to the setting of smoothly overlapping 3-branes by postulating that each brane carries its own field theory for the matter degrees of freedom, and that free motion of those degrees of freedom corresponds to free fall along the geodesics in their own brane. We do not allow for particle exchange between the branes, because in that case we should rather consider a single brane having non-trivial topology and carrying a single field theory for the matter degrees of freedom.

The second 3-brane will carry its own $U(1)$ gauge symmetry and charged particles with charges $\tilde{q}_{J}$ and masses $\tilde{m}_{J}$. The corresponding fields are $\tilde{\psi}_{J}$ and $\tilde{A}_{\mu}$, and the corresponding action is

$S_{2}[\tilde{\psi}, \tilde{A}]=\int d^{4} \tilde{x} \mathcal{L}(\tilde{\psi}, \tilde{A})$

How could crosstalk work? The simplest (but still interesting) models would assume Yukawa interactions involving scalar particles if we wish to stay within the framework of renormalizable models. However, here we assume that our electrons and protons can see the photons from the second brane in those volumes where the branes overlap. Renormalizability implies that the coupling of the charged particles on our brane to the photons from the second brane in the overlap region $V_{12}$ is

$S_{12}[\psi, \tilde{A}]=\int d t \int_{V_{12}} d^{3} x \sum_{I} g_{I} \bar{\psi}_{I} \gamma^{\mu} \tilde{A}_{\mu} \psi_{I}$,

and there is a corresponding equation for the coupling $S_{21}[\tilde{\psi}, A]$ of our photons to the fermions from the second brane. Note the universality of the propagation speed of the $U$ (1) gauge fields on both branes, because the free equations of motion for both kinds of photons in the overlap region are $\partial_{\mu} F^{\mu \nu}=0, \quad \partial_{\mu} \tilde{F}^{\mu v}=0$.

In the overlap region, the $U(1)$ from the second brane would enhance our own $U(1)$ symmetry to $U(1) \times U(1)$,

$\psi_{I}^{\prime}(x)=\exp \left(\mathrm{i} q_{I} f(x)+\mathrm{i} g_{I} \tilde{f}(x)\right) \psi_{I}(x)$,

$A_{\mu}^{\prime}(x)=A_{\mu}(x)+\partial_{\mu} f(x), \quad \tilde{A}_{\mu}^{\prime}(x)=\tilde{A}_{\mu}(x)+\partial_{\mu} \tilde{f}(x)$.

The onset of the additional $U(1)$ couplings at the boundary $\partial V_{12}$ of the overlap region generates steplike discontinuities in the equations of motion but no $\delta$ function terms, since the discontinuities enter only through the $\partial \mathcal{L} / \partial A_{\mu}$ and $\partial \mathcal{L} / \partial \bar{\psi}_{I}$ terms in the Lagrange equations.

Note that due to the lack of restrictions on $U(1)$ gauge couplings, electromagnetic crosstalk between overlapping branes appears like a natural and generic possibility if both branes carry $U(1)$ gauge theories. The same cannot be said about non-abelian crosstalk, since the gauge transformations for a non-abelian gauge field,

$A_{\mu}^{\prime}=U \cdot A_{\mu} \cdot U^{-1}+\frac{\mathrm{i}}{q} U \cdot \partial_{\mu} U^{-1}$ 
require universality of the gauge coupling $q$ for the nonabelian group. Therefore, while the two branes would not need to carry the same sets of representations of a nonabelian gauge group for corresponding crosstalk, non-abelian crosstalk coupling constants would be restricted by the requirements

$g=\tilde{q}, \quad \tilde{g}=q$.

Calculating the energy-momentum tensor for $S=S_{1}+$ $S_{2}+S_{12}+S_{21}$ in Coulomb gauge (see e.g. Sect. 21.4 in Ref. [15]) yields the Coulomb interaction terms in the Hamiltonian in the overlap region,

$$
\begin{aligned}
H_{11}= & \sum_{I I^{\prime}}\left(q_{I} q_{I^{\prime}}+g_{I} g_{I^{\prime}}\right) \int_{V_{12}} d^{3} \boldsymbol{x} \int d^{3} \boldsymbol{x}^{\prime} \\
& \times \sum_{s s^{\prime}} \frac{\psi_{I s}^{+}(\boldsymbol{x}) \psi_{I^{\prime} s^{\prime}}^{+}\left(\boldsymbol{x}^{\prime}\right) \psi_{I^{\prime} s^{\prime}}\left(\boldsymbol{x}^{\prime}\right) \psi_{I s}(\boldsymbol{x})}{8 \pi\left|\boldsymbol{x}-\boldsymbol{x}^{\prime}\right|}, \\
H_{12}= & \sum_{I J}\left(q_{I} \tilde{g}_{J}+g_{I} \tilde{q}_{J}\right) \int_{V_{12}} d^{3} \boldsymbol{x} \int d^{3} \boldsymbol{x}^{\prime} \\
& \times \sum_{s s^{\prime}} \frac{\psi_{I S}^{+}(\boldsymbol{x}) \tilde{\psi}_{J s^{\prime}}^{+}\left(\boldsymbol{x}^{\prime}\right) \tilde{\psi}_{J s^{\prime}}\left(\boldsymbol{x}^{\prime}\right) \psi_{I s}(\boldsymbol{x})}{4 \pi\left|\boldsymbol{x}-\boldsymbol{x}^{\prime}\right|}
\end{aligned}
$$

and a corresponding term $\mathrm{H}_{22}$ for the internal Coulomb interactions in the second brane. Here we used the Schrödinger picture field operators $\psi_{I s}(\boldsymbol{x})$ and $s, s^{\prime}$ are Dirac labels. Superficially, (6) always looks repulsive between Dirac fields of the same flavor, but recall that the actual particle and antiparticle creation operators are $b_{s}^{+}(\boldsymbol{k})$ and $d_{s}^{+}(\boldsymbol{k})$, respectively. Substituting the mode expansions $\psi \sim b+d^{+}$and normal ordering leads to the attractive Coulomb terms between particles and their anti-particles.

The effective modification of Coulomb interactions between charged particles on our brane has all kinds of interesting possible consequences. Everyday physics as we know it could be strongly modified in the overlap region. The electrostatic repulsion between electrons or protons would increase according to $e^{2} \rightarrow e^{2}+g_{e}^{2}$ and $e^{2} \rightarrow e^{2}+g_{p}^{2}$, respectively. The effective coupling constant between electrons and protons would change from $-e^{2}$ to $-e^{2}+g_{e} g_{p}$. Hydrogen atoms could be weaker or more strongly bound, or they are not bound at all if

$-e^{2}+g_{e} g_{p}>0$.

In this case, positrons could bind with protons because charge conjugation still applies to the Dirac equations in the overlap region and therefore $g_{\bar{e}}=-g_{e}$.

The term (7) would allow for the formation of exotic bound states of particles from the two branes. Furthermore, if we assume matter/anti-matter asymmetry also on the second brane, the Coulomb term (7) seems to favor electromagnetic attraction between the branes if
$\sum_{I J}\left(q_{I} \tilde{g}_{J}+g_{I} \tilde{q}_{J}\right)=\sum_{I} g_{I} \sum_{J} \tilde{q}_{J}<0$,

and electromagnetic repulsion if $\sum_{I} g_{I} \sum_{J} \tilde{q}_{J}>0$. Here we used the fact that the sum over charges of non-confined low-energy particle states in our brane vanishes, $\sum_{I} q_{I}=$ $q_{e}+q_{p}=0$.

This leaves a lot of interesting possible implications of 3-brane overlap. However, except for the particular case $e^{2}-g_{e} g_{p}=0$, there will be hydrogen type bound states of particles with reduced mass $\mu=m_{e} m_{p} /\left(m_{e}+m_{p}\right)$ in the overlap region, albeit with a potentially very different effective fine structure constant

$\alpha_{12}=\left|e^{2}-g_{e} g_{p}\right| / 4 \pi$.

The energy levels of these hydrogen type atoms are therefore shifted in leading order according to $E_{12, n}=\left(\alpha_{12} / \alpha\right)^{2} E_{n}$, which implies a corresponding shift in the emitted or absorbed wavelengths,

$\lambda_{12}=\frac{e^{4}}{\left(e^{2}-g_{e} g_{p}\right)^{2}} \lambda$.

The apparent redshift of the overlap region would therefore be

$z_{12}=(1+z) \frac{\lambda_{12}}{\lambda}-1=\frac{z e^{4}+2 e^{2} g_{e} g_{p}-g_{e}^{2} g_{p}^{2}}{\left(e^{2}-g_{e} g_{p}\right)^{2}}$,

or in the case of very weak inter-brane gauge couplings, $\left|g_{e} g_{p}\right| \ll e^{2}$,

$z_{12} \simeq z+2(1+z) \frac{g_{e} g_{p}}{e^{2}}$.

If the second brane would carry a gauge group $U(1)^{\otimes n}$, the crosstalk parameter $g_{e} g_{p}$ would apparently have the form $\sum_{i=1}^{n} g_{e}^{(i)} g_{p}^{(i)}$.

We have

$z_{12}>z \Leftrightarrow 0<g_{e} g_{p}<2 e^{2}$.

The observational signature of a brane overlap region at a redshift $z \leq 6$ would be a distortion of redshift binnings of hydrogen type clouds in the direction of the overlap region. For Lyman forests from quasars at $z \lesssim 6$ the signature would be a thinning of absorption lines in the range of the actual redshift parameter $z$ of the overlap region $V_{12}$, accompanied by a higher intensity of absorption lines at higher redshift or at lower redshift depending on whether the inequalities in (11) hold or not. If the overlap region is near the onset of the Gunn-Peterson trough, it can delay or advance the apparent onset of the trough in the direction of $V_{12}$, i.e. reionization would appear to have occurred earlier or later in the direction of $V_{12}$ than in other directions in our 3-brane. 


\section{Other implications}

Electromagnetic crosstalk increases the repulsion between like particles and can weaken or strengthen the electromagnetic coupling between electrons and protons, depending on $g_{e} g_{p}$ (11). This also implies that Bremsstrahlung emission into ordinary photons is weaker or stronger in $V_{12}$, since the emission probability will be proportional to $\alpha_{12}^{2} \alpha$. However, the total Bremsstrahlung emission probability from electrons into both kinds of photons will be proportional to $\alpha_{12}^{2}\left[\alpha+\left(g_{e}^{2} / 4 \pi\right)\right]$, and the same proportionality also holds for dipole emission from atomic transitions. This implies that we get weaker total electromagnetic emission in a smaller $g_{e} g_{p}$ range than the range in condition (11),

$$
\begin{aligned}
& P_{\gamma+\tilde{\gamma}, 12}<P_{\gamma} \\
& \Leftrightarrow e^{2}-\frac{e^{3}}{\sqrt{e^{2}+g_{e}^{2}}}<g_{e} g_{p}<e^{2}+\frac{e^{3}}{\sqrt{e^{2}+g_{e}^{2}}} .
\end{aligned}
$$

We expect electromagnetic cooling of contracting gas clouds to be less efficient for $P_{\gamma+\tilde{\gamma}, 12}<P_{\gamma}$ and more efficient otherwise. An increased mass density in an overlap region yields stronger curved geodesics, but it does not help with the formation of stars and galaxies if cooling is suppressed. Therefore we would expect slower formation of stars and galaxies in an overlap region where the inequalities in (12) hold, and accelerated formation otherwise. The effect on structure formation should have the following consequences for the observed perturbation of absorption lines at the redshift $z$ of the region $V_{12}$ :

If the inequalities in (11) do not hold, the apparent redshift $z_{12}$ would satisfy $z_{12}<z$, and there could be more hydrogen clouds with higher column densities in the overlap region due to $P_{\gamma+\tilde{\gamma}, 12}>P_{\gamma}$. The thinning out of absorption lines at $z$ should be there, but the increase at $z_{12}<z$ would be more pronounced than from the redshift effect (10) alone.

On the other hand, if the inequalities in (12) hold, the apparent redshift $z_{12}$ would satisfy $z_{12}>z$, and there might also be fewer hydrogen clouds with smaller column densities in the overlap region. The thinning out of absorption lines at $z$ should be there, but the increase at $z_{12}>z$ would be less pronounced. Please note that this scenario of reduced radiative cooling due to brane overlap could also help with the problem of overcooling in star formation histories; see e.g. [16] and references therein for a discussion of the overcooling problem.

As pointed out in Sect. 2, the primary observational effect of electromagnetic crosstalk between branes should be depletion of signals at the redshift $z$ of the overlap region $V_{12}$ and increase of signals at the redshift $z_{12}$ (10). Radiation sources in $V_{12}$ would then be assigned to higher or lower redshift values, depending on $g_{e} g_{p}$. In terms of visible radiation signals, a large brane overlap region $V_{12}$ would then appear as a dark trough in front or behind an apparent wall, or as a dark channel in front or behind an apparent filament. Whether the observed superlarge structures at $z \sim 1.3$ [17] or $1.6<z<2.1$ [18] could be explained by brane crosstalk would then depend on successful correlation with corresponding perturbations in the Lyman $\alpha$ forest. The discovery of these superlarge structures could herald the dawn of brane astronomy.

\section{Conclusions}

Equation (6) shows that crosstalk between gauge theories in overlapping branes affords local gauge couplings without promoting the couplings themselves to dynamical fields. This should impact the redshift distribution of Lyman $\alpha$ absorption lines by suppressing absorption lines at the redshift $z$ of the brane overlap region while increasing intensity of absorption lines at higher or lower redshift $z_{12}$ (10), depending on the electromagnetic crosstalk parameter $g_{e} g_{p}$. The redshift distortion from overlapping branes can also generate apparent large scale structure on scales that would violate size limits from structure formation in an isolated evolving 3-brane, thus explaining the possible absence of an "End of Greatness".

It is known since 1971 that quasars shine light on the intergalactic medium. Maybe quasars shine light on branes, too.

Acknowledgments This work was supported by NSERC Canada and by the Perimeter Institute for Theoretical Physics. Research at Perimeter Institute is supported by the Government of Canada through Industry Canada and by the Province of Ontario through the Ministry of Economic Development and Innovation.

Open Access This article is distributed under the terms of the Creative Commons Attribution License which permits any use, distribution, and reproduction in any medium, provided the original author(s) and the source are credited.

Funded by SCOAP 3 / License Version CC BY 4.0.

\section{References}

1. T. Kaluza, Sitzungsber. Preuss. Akad. Wiss. Jg. 1921 (Jul.-Dez.), $966(1921)$

2. O. Klein, Z. Phys. 37, 59 (1926)

3. G.R. Dvali, G. Gabadadze, M. Porrati, Phys. Lett. B 485, 208 (2000)

4. G.R. Dvali, G. Gabadadze, Phys. Rev. D 63, 065007 (2001)

5. C. de Rham, S. Hofmann, J. Khoury, A.J. Tolley, JCAP 0802, 011 (2008)

6. C. de Rham, J. Khoury, A.J. Tolley, Phys. Rev. Lett. 103, 161601 (2009)

7. C. Deffayet, Phys. Lett. B 502, 199 (2001)

8. C. Deffayet, G.R. Dvali, G. Gabadadze, Phys. Rev. D 65, 044023 (2002)

9. M.A. Luty, M. Porrati, R. Rattazzi, JHEP 0309, 029 (2003)

10. A. Nicolis, R. Rattazzi, E. Trincherini, Phys. Rev. D 79, 064036 (2009) 
11. R. Dick, Class. Quantum Grav. 18, R1 (2001)

12. R. Dick, Acta Phys. Pol. B 32, 3669 (2001)

13. A. Lue, Phys. Rep. 423, 1 (2006)

14. R. Cordero, A. Vilenkin, Phys. Rev. D 65, 083519 (2002)

15. R. Dick, Advanced Quantum Mechanics-Materials and Photons (Springer, New York, 2012)

16. I.G. McCarthy, J. Schaye, R.G. Bower, T.J. Ponman, C.M. Booth, C. Dalla, Vecchia, V. Springel, Mon. Not. R. Astron. Soc. 412, 1965 (2011)
17. R.G. Clowes, K.A. Harris, S. Raghunathan, L.E. Campusano, I.K. Söchting, M.J. Graham, Mon. Not. R. Astron. Soc. 429, 2910 (2013)

18. I. Horváth, J. Hakkila, Z. Bagoly, Astron. Astrophys. 561, L12 (2014) 